\title{
ON STRONGLY ORTHOGONAL MARTINGALES IN UMD BANACH SPACES
}

\author{
BY
}

IVAN S. YAROSLAVTSEV (LEIPZIG)

\begin{abstract}
In the present paper we introduce the notion of strongly orthogonal martingales. Moreover, we show that for any UMD Banach space $X$ and for any $X$-valued strongly orthogonal martingales $M$ and $N$ such that $N$ is weakly differentially subordinate to $M$, one has, for all $1<p<\infty$,

$$
\mathbb{E}\left\|N_{t}\right\|^{p} \leqslant \chi_{p, X}^{p} \mathbb{E}\left\|M_{t}\right\|^{p}, \quad t \geqslant 0
$$

with the sharp constant $\chi_{p, X}$ being the norm of a decoupling-type martingale transform and lying in the range

$$
\begin{aligned}
\max \left\{\sqrt{\beta_{p, X}}, \sqrt{\hbar_{p, X}}\right\} & \leqslant \max \left\{\beta_{p, X}^{\gamma,+}, \beta_{p, X}^{\gamma,-}\right\} \\
& \leqslant \chi_{p, X} \leqslant \min \left\{\beta_{p, X}, \hbar_{p, X}\right\}
\end{aligned}
$$

where $\beta_{p, X}$ is the $\mathrm{UMD}_{p}$ constant of $X, \hbar_{p, X}$ is the norm of the Hilbert transform on $L^{p}(\mathbb{R} ; X)$, and $\beta_{p, X}^{\gamma,+}$ and $\beta_{p, X}^{\gamma,-}$ are the Gaussian decoupling constants.
\end{abstract}

2020 Mathematics Subject Classification: Primary 60G44, 60H05; Secondary 60B11, 32U05.

Key words and phrases: strongly orthogonal martingales, weak differential subordination, UMD, sharp estimates, decoupling constant, martingale transform, Hilbert transform, diagonally plurisubharmonic function.

\section{INTRODUCTION}

Weak differential subordination of Banach-space-valued martingales was recently discovered in [36], [37], [35], [24] as a natural extension of differential subordination in the sense of Burkholder and Wang [32], [8] to infinite dimensions, and it has the following form: for a given Banach space $X$, an $X$-valued martingale $N$ is weakly differentially subordinate to an $X$-valued local martingale $M$ if a.s.

$$
\begin{aligned}
\left|\left\langle N_{0}, x^{*}\right\rangle\right| & \leqslant\left|\left\langle M_{0}, x^{*}\right\rangle\right|, \\
{\left[\left\langle N, x^{*}\right\rangle\right]_{t}-\left[\left\langle N, x^{*}\right\rangle\right]_{s} } & \leqslant\left[\left\langle M, x^{*}\right\rangle\right]_{t}-\left[\left\langle M, x^{*}\right\rangle\right]_{s}, \quad 0 \leqslant s \leqslant t,
\end{aligned}
$$


for any $x^{*} \in X^{*}$, where $[\cdot]$ is the quadratic variation of a martingale (see Subsection 2.2.

Weak differential subordination, especially if $X$ satisfies the UMD property (see Subsection 2.1), has several applications in harmonic analysis. On the one hand, $L^{p}$-bounds for weakly differentially subordinate purely discontinuous martingales imply estimates for $L^{p}$-norms of Lévy multipliers. Namely, it was shown in [36] that if $T_{m}$ is a Lévy multiplier (i.e. a Fourier multiplier generated by a Lévy measure [2], [1]), then by using weakly differentially subordinate purely discontinuous martingales one finds that for any $1<p<\infty$ the $L^{p}$-norm of $T_{m}$ acting on $X$-valued functions is bounded by the UMD constant $\beta_{p, X}$ (whose finiteness characterizes the UMD property, see Subsection 2.1.

On the other hand, various bounds for weakly differentially subordinate orthogonal martingales coincide with the same type of estimates for the Hilbert transform (see [24] by Osękowski and the present author). Recall that two $X$-valued martingales $M$ and $N$ are orthogonal if for any $x^{*} \in X^{*}$ one has a.s.,

$$
\left\langle M_{0}, x^{*}\right\rangle \cdot\left\langle N_{0}, x^{*}\right\rangle=0 \quad \text { and } \quad\left[\left\langle M, x^{*}\right\rangle,\left\langle N, x^{*}\right\rangle\right]_{t}=0, \quad t \geqslant 0,
$$

where $[\cdot, \cdot]$ is the covariation of two martingales (see Subsection 2.2). In particular, it was shown in [24] that for any UMD Banach space $X$ and any $X$-valued orthogonal martingales $M$ and $N$ such that $N$ is weakly differentially subordinate to $M$, for every $1<p<\infty$ one has

$$
\mathbb{E}\left\|N_{t}\right\|^{p} \leqslant \hbar_{p, X}^{p} \mathbb{E}\left\|M_{t}\right\|^{p}, \quad t \geqslant 0,
$$

where the sharp constant $\hbar_{p, X}$ is the norm of the Hilbert transform on $L^{p}(\mathbb{R} ; X)$.

The goal of the present paper is to prove sharp $L^{p}$-estimates for strongly orthogonal weakly differentially subordinate martingales. We call two $X$-valued martingales $M$ and $N$ strongly orthogonal if for any $x^{*}, y^{*} \in X^{*}$ a.s.

$$
\left\langle M_{0}, x^{*}\right\rangle \cdot\left\langle N_{0}, y^{*}\right\rangle=0 \quad \text { and }\left[\left\langle M, x^{*}\right\rangle,\left\langle N, y^{*}\right\rangle\right]_{t}=0, \quad t \geqslant 0 .
$$

A classical example of strongly orthogonal martingales is provided by stochastic integrals $\int \Phi \mathrm{d} W$ and $\int \Phi \mathrm{d} \widetilde{W}$, where $\Phi$ is an $X$-valued elementary predictable process, and $W$ and $\widetilde{W}$ are independent Brownian motions. We prove that for any strongly orthogonal weakly differentially subordinate martingales $M$ and $N$,

$$
\mathbb{E}\left\|N_{t}\right\|^{p} \leqslant \chi^{p} \mathbb{E}\left\|M_{t}\right\|^{p}, \quad t \geqslant 0,1<p<\infty,
$$

where the sharp constant $\chi=\chi_{p, X}$ is within the range

$$
\max \left\{\sqrt{\beta_{p, X}}, \sqrt{\hbar_{p, X}}\right\} \leqslant \chi_{p, X} \leqslant \min \left\{\beta_{p, X}, \hbar_{p, X}\right\} .
$$

The main technique we use in order to prove (1.1) is the Bellman function method. More specifically, we show that the following are equivalent: 
(A) (1.1) holds for a constant $\chi>0$,

(B) there exists $U^{S O}: X+i X \rightarrow \mathbb{R}$ such that $U^{S O}(x) \geqslant 0$ for any $x \in X$, $z \mapsto U^{S O}\left(x_{0}+i y_{0}+z x\right)$ is subharmonic in $z \in \mathbb{C}$ for any $x_{0}, y_{0}, x \in X$, and

$$
U^{S O}(x+i y) \leqslant \chi^{p}\|x\|^{p}-\|y\|^{p}, \quad x, y \in X .
$$

Notice that this method is not new. Namely, in [36] the Burkholder function $U$ : $X \times X \rightarrow \mathbb{R}$ was applied which first appeared in the paper [9] by Burkholder, and in [24] a plurisubhirmonic function $U_{\mathcal{H}}: X+i X \rightarrow \mathbb{R}$ was used, which was first constructed in the paper [17] by Hollenbeck, Kalton, and Verbitsky. The novelty of the present paper is in minimizing the necessary properties of the Bellman function. Namely, both $-U$ and $U_{\mathcal{H}}$ satisfy the property (B) above (which makes the upper bound of (1.2) elementary).

In order to show the lower bounds of $(1.2)$ and to characterize the least admissible constant $\chi_{p, X}$ we will need the example presented above. It is shown in Sections 3 and 4 that the sharp constant $\chi_{p, X}$ is the smallest constant $\chi>0$ such that for any independent Brownian motions $W$ and $\widetilde{W}$ and for any elementary predictable $X$-valued $\Phi$ one has

$$
\mathbb{E}\left\|\int_{0}^{\infty} \Phi \mathrm{d} \widetilde{W}\right\|^{p} \leqslant \chi^{p} \mathbb{E}\left\|\int_{0}^{\infty} \Phi \mathrm{d} W\right\|^{p} .
$$

Thus the desired lower bound of (1.2) follows from the well-known decouplingtype inequalities of Garling [13].

Notice that if $X=\mathbb{R}$, then $\chi_{p, X}=\hbar_{p, X}$ (see Remark 3.3). Nevertheless, it remains open whether this equality holds for a general UMD Banach space $X$. Moreover, if this is the case, then it solves a celebrated open problem about linear dependence of the constants $\beta_{p, X}$ and $\hbar_{p, X}$; see [6, p. 48] and [18], [15], [36], [24] (so far only a quadratic dependence is known, see (2.2)).

\section{PRELIMINARIES}

Throughout the paper all Banach spaces are assumed to be over $\mathbb{R}$ unless otherwise stated. We also assume that any filtration satisfies the usual conditions. In particular, any filtration is right-continuous, and thus all the local martingales exploited in the article have càdlàg versions (right continuous with left limits [28], [36]). Furthermore, for any Banach space $X$, for any càdlàg process $A: \mathbb{R}_{+} \times \Omega \rightarrow X$ and any stopping time $\tau$ we define

$$
\Delta A_{\tau}:=\lim _{\varepsilon \rightarrow 0}\left(A_{\tau}-A_{(\tau-\varepsilon) \vee 0}\right) .
$$

2.1. UMD Banach spaces. A Banach space $X$ is called $U M D$ if for some (equivalently, all) $p \in(1, \infty)$ there exists a constant $\beta>0$ such that for every $N \geqslant 1$, 
every martingale difference sequence $\left(d_{n}\right)_{n=1}^{N}$ in $L^{p}(\Omega ; X)$, and every $\{-1,1\}$ valued sequence $\left(\varepsilon_{n}\right)_{n=1}^{N}$ we have

$$
\left(\mathbb{E}\left\|\sum_{n=1}^{N} \varepsilon_{n} d_{n}\right\|^{p}\right)^{1 / p} \leqslant \beta\left(\mathbb{E}\left\|\sum_{n=1}^{N} d_{n}\right\|^{p}\right)^{1 / p} .
$$

The least admissible constant $\beta$ is denoted by $\beta_{p, X}$ and called the $U M D_{p}$ constant or, if the value of $p$ is understood, the UMD constant of $X$. It is well-known that UMD spaces have a large number of useful properties, such as being reflexive. Examples of UMD spaces include all finite-dimensional spaces and the reflexive range of $L^{q}$-, Besov, Sobolev, Schatten class, and Musielak-Orlicz spaces. Examples of spaces without the UMD property include all nonreflexive Banach spaces, e.g. $L^{1}(0,1)$ or $C([0,1])$. We refer to [10], [18], [27], [25] for details.

2.2. Quadratic variation. Let $(\Omega, \mathcal{F}, \mathbb{P})$ be a probability space with a filtration $\mathbb{F}=\left(\mathcal{F}_{t}\right)_{t \geqslant 0}$ that satisfies the usual conditions. Let $M: \mathbb{R}_{+} \times \Omega \rightarrow \mathbb{R}$ be a local martingale. We define the quadratic variation of $M$

$$
[M]_{t}:=\mathbb{P}-\lim _{\operatorname{mesh} \rightarrow 0} \sum_{n=1}^{N}\left|M\left(t_{n}\right)-M\left(t_{n-1}\right)\right|^{2},
$$

where the limit in probability is taken over partitions $0=t_{0}<\cdots<t_{N}=t$. Note that $[M]$ exists and is nondecreasing a.s. The reader can find more on quadratic variations in [20], [26], [12]. For any martingales $M, N: \mathbb{R}_{+} \times \Omega \rightarrow \mathbb{R}$ we can define their covariation $[M, N]: \mathbb{R}_{+} \times \Omega \rightarrow \mathbb{R}$ as $[M, N]:=\frac{1}{4}([M+N]-$ $[M-N])$. Since $M$ and $N$ have càdlàg versions, $[M, N]$ has a càdlàg version as well (see e.g. [19, Theorem I.4.47]).

A local martingale $M: \mathbb{R}_{+} \times \Omega \rightarrow \mathbb{R}$ is called purely discontinuous if $[M]$ is a.s. pure jump, i.e. $[M]_{t}=\sum_{0 \leqslant s \leqslant t} \Delta[M]_{s}$ a.s. Let $X$ be a Banach space. Then an $X$-valued local martingale $M: \mathbb{R}_{+} \times \Omega \rightarrow X$ is called purely discontinuous if $\left\langle M, x^{*}\right\rangle$ is purely discontinuous for any $x^{*} \in X^{*}$. Note that if $X$ is UMD, then any local martingale $M$ has a unique decomposition into the sum of a continuous local martingale $M^{c}$ with $M_{0}^{c}=0$ and a purely discontinuous local martingale $M^{d}$ (see [33]). We refer to [20], [19], [36], [37], [33] for details on purely discontinuous martingales.

2.3. Weak differential subordination of martingales. Let $X$ be a Banach space. Let $M, N: \mathbb{R}_{+} \times \Omega \rightarrow X$ be local martingales. Then we say that $N$ is weakly differentially subordinate to $M$ (written $N \stackrel{w}{\ll} M$ ) if for each $x^{*} \in X^{*}$, [ $\left.\left\langle M, x^{*}\right\rangle\right]-$ $\left[\left\langle N, x^{*}\right\rangle\right]$ is an a.s. nondecreasing function and $\left|\left\langle N_{0}, x^{*}\right\rangle\right| \leqslant\left|\left\langle M_{0}, x^{*}\right\rangle\right|$ a.s.

This definition first appeared in [36] as a natural extension of differential subordination of real-valued martingales. Later in [37] the first $L^{p}$-estimates for weakly differentially subordinate martingales were obtained, which have been significantly improved in [24] in the continuous-time case. 
2.4. Orthogonal martingales. Let $M$ and $N$ be local martingales taking values in a given Banach space $X$. Then $M$ and $N$ are said to be orthogonal if $\left\langle M_{0}, x^{*}\right\rangle$. $\left\langle N_{0}, x^{*}\right\rangle=0$ and $\left[\left\langle M, x^{*}\right\rangle,\left\langle N, x^{*}\right\rangle\right]=0$ almost surely for all $x^{*} \in X^{*}$.

REMARK 2.1. Assume that $M$ and $N$ are local martingales taking values in some Banach space $X$. If $M$ and $N$ are orthogonal and $N$ is weakly differentially subordinate to $M$, then $N_{0}=0$ almost surely (which follows immediately from the above definitions, see [24]). Moreover, under these assumptions, $N$ must have continuous trajectories with probability 1 . Indeed, in that case for any fixed $x^{*} \in X^{*}$ the real-valued local martingales $\left\langle M, x^{*}\right\rangle$ and $\left\langle N, x^{*}\right\rangle$ are orthogonal and $\left\langle N, x^{*}\right\rangle \ll\left\langle M, x^{*}\right\rangle$. Therefore, $\left\langle N, x^{*}\right\rangle$ has a continuous version for each $x^{*} \in X^{*}$ by [23, Lemma 3.1] (see also [4, Lemma 1]), which in turn implies that $N$ is continuous since any $X$-valued local martingale has a càdlàg version.

2.5. Stochastic integration. For given Banach spaces $X$ and $Y, \mathcal{L}(X, Y)$ will denote the class of all linear operators from $X$ to $Y$. We will also write $\mathcal{L}(X)=$ $\mathcal{L}(X, X)$. Suppose that $H$ is a Hilbert space. For each $h \in H$ and $x \in X$, we denote by $h \otimes x$ the associated linear operator given by $g \mapsto\langle g, h\rangle x, g \in H$. A process $\Phi: \mathbb{R}_{+} \times \Omega \rightarrow \mathcal{L}(H, X)$ is called elementary predictable with respect to the filtration $\mathbb{F}=\left(\mathcal{F}_{t}\right)_{t \geqslant 0}$ if it is of the form

$$
\Phi(t, \omega)=\sum_{k=1}^{K} \sum_{m=1}^{M} \mathbf{1}_{\left(t_{k-1}, t_{k}\right] \times B_{m k}}(t, \omega) \sum_{n=1}^{N} h_{n} \otimes x_{k m n}, \quad t \geqslant 0, \omega \in \Omega .
$$

Here $0 \leqslant t_{0}<\cdots<t_{K}<\infty$, the sets $B_{1 k}, \ldots, B_{M k}$ belong to $\mathcal{F}_{t_{k-1}}$ for each $k=1, \ldots, K$, and the vectors $h_{1}, \ldots, h_{N}$ are assumed to be orthogonal. Suppose further that $M$ is an adapted local martingale taking values in $H$. Then the stochastic integral $\int \Phi \mathrm{d} M: \mathbb{R}_{+} \times \Omega \rightarrow X$ of $\Phi$ with respect to $M$ is defined by the formula

$$
\int_{0}^{t} \Phi \mathrm{d} M=\sum_{k=1}^{K} \sum_{m=1}^{M} \mathbf{1}_{B_{m k}} \sum_{n=1}^{N}\left\langle\left(M\left(t_{k} \wedge t\right)-M\left(t_{k-1} \wedge t\right)\right), h_{n}\right\rangle x_{k m n}, \quad t \geqslant 0 .
$$

REMARK 2.2. If both $X$ and $H$ are finite-dimensional, then we may assume that $X$ is isomorphic to $\mathbb{R}^{d}$, and thus by [20, Theorems 26.6 and 26.12] we can extend the stochastic integration from elementary predictable processes to all the predictable processes $\Phi: \mathbb{R}_{+} \times \Omega \rightarrow \mathcal{L}(H, X)$ with

$$
\mathbb{E}\left(\sum_{i=1}^{n} \int_{0}^{\infty}\left\|\Phi h_{i}\right\|^{2} \mathrm{~d}\left[\left\langle M, h_{i}\right\rangle\right]_{s}\right)^{1 / 2}<\infty
$$

where $n$ is the dimension of $H$ and $h_{1}, \ldots, h_{n}$ is an orthonormal basis of $H$. In fact, a similar characterization of stochastic integration can be shown for infinitedimensional $X$ and $H$ by using $\gamma$-norms (see [22], [34], [31], [29]). 
2.6. Hilbert transform. Let $X$ be a Banach space. The Hilbert transform $\mathcal{H}_{X}$ is the singular integral operator that maps a step function $f: \mathbb{R} \rightarrow X$ to the function

$$
\left(\mathcal{H}_{X} f\right)(t):=\frac{1}{\pi} \text { p.v. } \int_{\mathbb{R}} \frac{f(s)}{t-s} \mathrm{~d} s, \quad t \in \mathbb{R} .
$$

For any $1<p<\infty$ we denote the norm of $\mathcal{H}_{X}$ on $L^{p}(\mathbb{R} ; X)$ by $\hbar_{p, X}$. Note that from [7], [5] we know that $\hbar_{p, X}<\infty$ if and only if $X$ is UMD. Moreover, by [13], [5], for every $1<p<\infty$,

$$
\sqrt{\beta_{p, X}} \leqslant \hbar_{p, X} \leqslant \beta_{p, X}^{2} .
$$

REMARK 2.3. Recently in [24] it was shown that $\hbar_{p, X}$ is the smallest constant $\hbar$ such that there exists a plurisubharmonic function $U_{\mathcal{H}}: X+i X \rightarrow \mathbb{R}$ (i.e. $z \mapsto$ $U_{\mathcal{H}}\left(x_{0}+i y_{0}+z(x+i y)\right)$ is subharmonic in $z \in \mathbb{C}$ for any fixed $\left.x_{0}, y_{0}, x, y \in X\right)$ such that $U_{\mathcal{H}}(x) \geqslant 0$ for any $x \in X$ and $U_{\mathcal{H}}(x+i y) \leqslant \hbar^{p}\|x\|^{p}-\|y\|^{p}$ for all $x, y \in X$.

2.7. Bellman functions and function approximation. Let $X$ be a UMD Banach space, and let $1<p<\infty$. Throughout the paper we will use various Bellman functions, which are functions $u: X \times X \rightarrow \mathbb{R}$ with appropriate properties. Let us list the functions we will use:

- the Burkholder function $U: X \times X \rightarrow \mathbb{R}$ (see e.g. [18] and the proof of Corollary 3.1 ,

- a plurisubharmonic function $U_{\mathcal{H}}: X+i X \rightarrow \mathbb{R}$ (see [24] and Subsection 2.6,

- a diagonally plurisubharmonic function $U^{S O}: X+i X \rightarrow \mathbb{R}$ (see Section 3 ).

For all these Bellman functions we may assume that $X$ is finite-dimensional and that the function is twice Fréchet differentiable by an approximation argument exploited in [37], [24], [3]. We will not repeat this argument here, but just briefly recall the main steps.

- Since $X$ is UMD, it is reflexive, and by the Pettis measurability theorem [18, Theorem 1.1.20] we may assume that $X$ is separable. Thus $X^{*}$ is separable as well, and there exists an increasing sequence $\left(Y_{n}\right)_{n \geqslant 1}$ of finite-dimensional subspaces of $X^{*}$ such that $X^{*}=\overline{\bigcup_{n} Y_{n}}$. Let $P_{n}: Y_{n} \rightarrow X^{*}$ be the inclusion operator. We will need that $\mathbb{E}\|\eta\|^{p} \leqslant c_{p, X}^{p} \mathbb{E}\|\xi\|^{p}$ for a certain pair of random variables $\xi, \eta \in L^{p}(\Omega ; X)$ and a certain constant $c_{p, X}$. Since $\left\|P_{n}^{*} x\right\| \nearrow\|x\|$ as $n \rightarrow \infty$ for any $x \in X$, by the monotone convergence theorem it is sufficient to show that $\mathbb{E}\left\|P_{n}^{*} \eta\right\|^{p} \leqslant c_{p, X}^{p} \mathbb{E}\left\|P_{n}^{*} \xi\right\|^{p}$ for any $n \geqslant 1$. Moreover, in fact we need to show that $\mathbb{E}\left\|P_{n}^{*} \eta\right\|^{p} \leqslant c_{p, Y_{n}^{*}}^{p} \mathbb{E}\left\|P_{n}^{*} \xi\right\|^{p}$ since in our case $c_{p, X}$ equals either $\beta_{p, X}, \hbar_{p, X}$, or $\chi_{p, X}$ (see Section 3 for the definition), and since all these constants can be represented 
as norms of operators having the same operators as their duals, analogously to [18, Proposition 4.2.17] one has $c_{p, X}=c_{p^{\prime}, X^{*}}\left(\right.$ where $p^{\prime}=p /(p-1)$ ), and in particular

$$
c_{p, Y_{n}^{*}}=c_{p^{\prime}, Y_{n}} \leqslant c_{p^{\prime}, X^{*}}=c_{p, X} .
$$

Thus it is sufficient to assume that $X$ is finite-dimensional since both $P_{n}^{*} \xi$ and $P_{n}^{*} \eta$ have their values in a finite-dimensional space $Y_{n}^{*}$.

- Since $X$ is finite-dimensional, for a Bellman function $u$ and for any $\varepsilon>0$ we can define $u_{\varepsilon}:=u * \varepsilon^{-1} \phi\left(\varepsilon^{-1} \cdot\right)$, where $\phi: X \times X \rightarrow \mathbb{R}_{+}$is a $C^{\infty}$ function with a compact support such that $\int_{X \times X} \phi(x, y) \mathrm{d} \lambda(x) \mathrm{d} \lambda(y)=1$ (here $\lambda$ is the Lebesgue measure on $X$, see e.g. [36, Remark 3.13] for the definition). Then $u_{\varepsilon}$ preserves such properties of $u$ as convexity, concavity, or subharmonicity on a linear subspace of $X \times X$, and $u_{\varepsilon} \rightarrow u$ as $\varepsilon \rightarrow 0$ locally uniformly on $X \times X$ by continuity of $u$. Therefore by this approximation argument we may assume that $u$ is $C^{\infty}$.

\section{THE $\chi_{p, X}$ CONSTANT}

Let $X$ be a Banach space, $1<p<\infty$. We define $\chi_{p, X} \in[0, \infty]$ to be the least number $\chi>0$ such that for any independent standard Brownian motions $W, \widetilde{W}: \mathbb{R}_{+} \times \Omega \rightarrow \mathbb{R}$ and for any elementary predictable process $\Phi: \mathbb{R}_{+} \times \Omega \rightarrow X$ with respect to the filtration generated by both $W$ and $\widetilde{W}$ one has

$$
\mathbb{E}\left\|\int_{0}^{\infty} \Phi \mathrm{d} \widetilde{W}\right\|^{p} \leqslant \chi^{p} \mathbb{E}\left\|\int_{0}^{\infty} \Phi \mathrm{d} W\right\|^{p}
$$

REMARK 3.1. $\chi_{p, X}$ can be equivalently defined in the following way. Let $\left(\gamma_{n}\right)_{n \geqslant 1}$ and $\left(\tilde{\gamma}_{n}\right)_{n \geqslant 1}$ be sequences of independent standard Gaussian random variables, $\mathcal{F}_{0}=\{\emptyset, \Omega\}$, and $\mathcal{F}_{n}=\sigma\left(\gamma_{1}, \tilde{\gamma}_{1}, \ldots, \gamma_{n}, \tilde{\gamma}_{n}\right)$ for $n \geqslant 1$. Then $\chi_{p, X}$ is the smallest $\chi>0$ such that for any $N \geqslant 1$ and any elementary step functions $v_{0}, \ldots, v_{N-1}: \Omega \rightarrow X$ with $v_{n}$ being $\mathcal{F}_{n}$-measurable for each $n=0, \ldots, N-1$, one has

$$
\mathbb{E}\left\|\sum_{n=1}^{N} \tilde{\gamma}_{n} v_{n-1}\right\|^{p} \leqslant \chi^{p} \mathbb{E}\left\|\sum_{n=1}^{N} \gamma_{n} v_{n-1}\right\|^{p}
$$

Indeed, one can represent the sums $\sum_{n=1}^{N} \gamma_{n} v_{n-1}$ and $\sum_{n=1}^{N} \tilde{\gamma}_{n} v_{n-1}$ as stochastic integrals with respect to independent Brownian motions $W$ and $\widetilde{W}$ by just letting $\gamma_{n}=W_{n}-W_{n-1}$ and $\tilde{\gamma}_{n}=\widetilde{W}_{n}-\widetilde{W}_{n-1}$. On the other hand, if $W$ and $\widetilde{W}$ are independent Brownian motions and if $\Phi$ is elementary predictable and defined by

$$
\Phi(t, \omega)=\sum_{k=1}^{K} \sum_{m=1}^{M} \mathbf{1}_{\left(t_{k-1}, t_{k}\right] \times B_{m k}}(t, \omega) x_{k m}, \quad t \geqslant 0, \omega \in \Omega,
$$


where $0 \leqslant t_{0}<\cdots<t_{K}<\infty$ and the sets $B_{1 k}, \ldots, B_{M k}$ belong to $\mathcal{F}_{t_{k-1}}$ for each $k=1, \ldots, K$, then one can represent the stochastic integrals $\Phi \cdot W$ and $\Phi \cdot \widetilde{W}$ as the following sums:

$$
\begin{aligned}
& \int_{0}^{\infty} \Phi \mathrm{d} W=\sum_{k=1}^{K} \sum_{m=1}^{M} \mathbf{1}_{B_{m k}}\left(W\left(t_{k}\right)-W\left(t_{k-1}\right)\right) x_{k m}=\sum_{k=1}^{K} v_{k-1} \gamma_{k}, \\
& \int_{0}^{\infty} \Phi \mathrm{d} \widetilde{W}=\sum_{k=1}^{K} \sum_{m=1}^{M} \mathbf{1}_{B_{m k}}\left(\widetilde{W}\left(t_{k}\right)-\widetilde{W}\left(t_{k-1}\right)\right) x_{k m}=\sum_{k=1}^{K} v_{k-1} \tilde{\gamma}_{k},
\end{aligned}
$$

where $\gamma_{k}=\frac{W\left(t_{k}\right)-W\left(t_{k-1}\right)}{\sqrt{t_{k}-t_{k-1}}}, \tilde{\gamma}_{k}=\frac{\widetilde{W}\left(t_{k}\right)-\widetilde{W}\left(t_{k-1}\right)}{\sqrt{t_{k}-t_{k-1}}}$, and

$$
v_{k-1}=\sqrt{t_{k}-t_{k-1}} \sum_{m=1}^{M} \mathbf{1}_{B_{m k}} x_{k m} .
$$

The martingale transform (3.1) appears when working with Volterra-type operators and stochastic shifts (see [16]).

Concerning the constant $\chi_{p, X}$ one can show the following proposition. First we will define diagonally plurisubharmonic functions.

DEFINITION 3.1. A function $F: X+i X \rightarrow \mathbb{R}$ is called diagonally plurisubharmonic if $z \mapsto F\left(x_{0}+i y_{0}+z x\right)$ is subharmonic in $z \in \mathbb{C}$ for any $x_{0}, y_{0}, x \in X$.

Proposition 3.1. Let $X$ be a Banach space, and let $1<p<\infty$. Then the following are equivalent:

(i) $\chi_{p, X}<\infty$,

(ii) there exists a constant $\chi>0$ and a diagonally plurisubharmonic $u: X+$ $i X \rightarrow \mathbb{R}$ such that $u(x) \geqslant 0$ for any $x \in X, x \mapsto u(x+i y)$ is convex in $x \in X$ for any $y \in X, y \mapsto u(x+i y)$ is concave in $y \in X$ for any $x \in X$, and

$$
u(x+i y) \leqslant \chi^{p}\|x\|^{p}-\|y\|^{p}, \quad x, y \in X .
$$

Moreover, in that case the smallest $\chi$ for which such a function $u$ exists equals $\chi_{p, X}$.

Proof. (i) $\Rightarrow$ (ii). We need to construct $u$ for $\chi=\chi_{p, X}$. Define

$$
\begin{aligned}
u(x+i y):=\inf \left\{\chi_{p, X}^{p} \mathbb{E}\left\|x+\int_{0}^{\infty} \Phi \mathrm{d} W\right\|^{p}-\mathbb{E}\left\|y+\int_{0}^{\infty} \Phi \mathrm{d} \widetilde{W}\right\|^{p}:\right. \\
\left.\Phi: \mathbb{R}_{+} \times \Omega \rightarrow X \text { elementary predictable }\right\}, \quad x, y \in X .
\end{aligned}
$$


First of all, $u$ is finite on $X+i X$, because for any elementary predictable $\Phi$ : $\mathbb{R}_{+} \times \Omega \rightarrow X$ and any $x, y \in X$, by the triangle inequality,

$$
\begin{aligned}
\chi_{p, X}^{p} \mathbb{E}\left\|x+\int_{0}^{\infty} \Phi \mathrm{d} W\right\|^{p}-\mathbb{E}\left\|y+\int_{0}^{\infty} \Phi \mathrm{d} \widetilde{W}\right\|^{p} \\
\quad \gtrsim p \chi_{p, X}^{p} \mathbb{E}\left\|\int_{0}^{\infty} \Phi \mathrm{d} W\right\|^{p}-\mathbb{E}\left\|\int_{0}^{\infty} \Phi \mathrm{d} \widetilde{W}\right\|^{p}-\chi_{p, X}^{p}\|x\|^{p}-\|y\|^{p} \\
\geqslant-\chi_{p, X}^{p}\|x\|^{p}-\|y\|^{p},
\end{aligned}
$$

where the last inequality holds by the definition of $\chi_{p, X}$.

Let us show that $u$ is continuous. For any $x, y, \tilde{x}, \tilde{y}$ one has, by the triangle inequality,

$$
\begin{aligned}
u(x+i y)= & \inf \left\{\chi_{p, X}^{p} \mathbb{E}\left\|x+\int_{0}^{\infty} \Phi \mathrm{d} W\right\|^{p}-\mathbb{E}\left\|y+\int_{0}^{\infty} \Phi \mathrm{d} \widetilde{W}\right\|^{p}:\right. \\
\left.\Phi: \mathbb{R}_{+} \times \Omega \rightarrow X \text { elementary predictable }\right\} & \\
\lesssim & \inf \left\{\chi_{p, X}^{p} \mathbb{E}\left\|\tilde{x}+\int_{0}^{\infty} \Phi \mathrm{d} W\right\|^{p}-\mathbb{E}\left\|\tilde{y}+\int_{0}^{\infty} \Phi \mathrm{d} \widetilde{W}\right\|^{p}:\right. \\
& \left.\Phi: \mathbb{R}_{+} \times \Omega \rightarrow X \text { elementary predictable }\right\} \\
& +\chi_{p, X}\|x-\tilde{x}\|^{p}+\|y-\tilde{y}\|^{p} \\
\leqslant & u(\tilde{x}+i \tilde{y})+\chi_{p, X}\|x-\tilde{x}\|^{p}+\|y-\tilde{y}\|^{p},
\end{aligned}
$$

and the continuity follows.

Now let us show that $u$ is diagonally plurisubharmonic. Fix $x_{0}, y_{0}, x \in X$. We need to show that $z \mapsto u\left(x_{0}+i y_{0}+z x\right)$ is subharmonic in $z \in \mathbb{C}$. To this end we need to prove that for any fixed $r>0$,

$$
u\left(x_{0}+i y_{0}\right) \leqslant \frac{1}{2 \pi} \int_{0}^{2 \pi} u\left(x_{0}+i y_{0}+x r e^{i \theta}\right) \mathrm{d} \theta .
$$

Let $W, \widetilde{W}: \mathbb{R}_{+} \times \Omega \rightarrow \mathbb{R}$ be independent standard Brownian motions. Define a stopping time $\tau$ by

$$
\tau:=\inf \left\{t \geqslant 0: W_{t}^{2}+\widetilde{W}_{t}^{2}=r\right\} .
$$

Fix $\varepsilon>0$. Since $u$ is continuous, there exist $\delta>0$ and a $\delta$-net $\left(a_{n}\right)_{n=1}^{N}=$ $\left(x_{n}+i y_{n}\right)_{n=1}^{N}$ of the compact set $A:=\left\{x_{0}+i y_{0}+x r e^{i \theta}: \theta \in[0,2 \pi)\right\} \subset X+i X$ with

$$
\left|u(a)-u\left(a_{n}\right)\right| \leqslant \varepsilon \quad \forall a \in A \text { such that }\left\|a-a_{n}\right\|<\delta
$$


(here the norm on $A$ is assumed to be the usual norm on $\mathbb{C}$ since $A$ can be represented as a circle on $\mathbb{C}$ ). Let $B_{t}:=W_{t+\tau}-W_{\tau}$ and $\widetilde{B}_{t}:=\widetilde{W}_{t+\tau}-\widetilde{W}_{\tau}$. Then $B$ and $\widetilde{B}$ are independent Brownian motions (see e.g. [20, Theorem 13.11]). Therefore by the definition of $u$ for every $n=1, \ldots, N$ there exists an elementary predictable process $\Phi_{n}: \mathbb{R}_{+} \rightarrow X$ with respect to the filtration generated by $B$ and $\widetilde{B}$ such that

$$
u\left(a_{n}\right) \geqslant \chi_{p, X}^{p} \mathbb{E}\left\|x_{n}+\int_{0}^{\infty} \Phi_{n} \mathrm{~d} B\right\|^{p}-\mathbb{E}\left\|y_{n}+\int_{0}^{\infty} \Phi_{n} \mathrm{~d} \widetilde{B}\right\|^{p}-\varepsilon .
$$

Now let us define a process $\Phi$ predictable with respect to the filtration generated by $W$ and $\widetilde{W}$ in the following way: $\Phi(t)=x$ if $t \leqslant \tau$ and $\Phi(t)=\Phi_{n}(t-\tau)$ if $t>\tau$ and $a_{n}$ is closest to $x_{0}+i y_{0}+x\left(W_{\tau}+i \widetilde{W}_{\tau}\right)$ among $\left(a_{n}\right)_{n=1}^{N}$. This is a predictable process and since $\Phi$ takes values in a finite-dimensional subspace of $X$, it can be approximated by an elementary predictable process (see Remark 2.2). Therefore

$$
\begin{aligned}
u\left(x_{0}+i y_{0}\right) \leqslant & \chi_{p, X}^{p} \mathbb{E}\left\|x_{0}+\int_{0}^{\infty} \Phi \mathrm{d} W\right\|^{p}-\mathbb{E}\left\|y_{0}+\int_{0}^{\infty} \Phi \mathrm{d} \widetilde{W}\right\|^{p} \\
= & \chi_{p, X}^{p} \mathbb{E}\left\|x_{0}+x W_{\tau}+\int_{0}^{\infty} \Phi(t) \mathrm{d} B_{t-\tau}\right\|^{p} \\
& -\mathbb{E}\left\|y_{0}+x \widetilde{W}_{\tau}+\int_{0}^{\infty} \Phi(t) \mathrm{d} \widetilde{B}_{t-\tau}\right\|^{p} \\
\stackrel{(\mathrm{i})}{=} & \frac{1}{2 \pi} \int_{0}^{2 \pi} \chi_{p, X}^{p} \mathbb{E}\left\|x_{0}+x \cos \theta+\int_{0}^{\infty} \Phi_{n(\theta)}(t) \mathrm{d} B_{t}\right\|^{p} \\
& -\mathbb{E}\left\|y_{0}+x \sin \theta+\int_{0}^{\infty} \Phi_{n(\theta)}(t) \mathrm{d} \widetilde{B}_{t}\right\|^{p} \mathrm{~d} \theta \\
\stackrel{(\text { ii) }}{\leqslant} & \frac{1}{2 \pi} \int_{0}^{2 \pi} \chi_{p, X}^{p} \mathbb{E}\left\|x_{n(\theta)}+\int_{0}^{\infty} \Phi_{n(\theta)}(t) \mathrm{d} B_{t}\right\|^{p} \\
& -\mathbb{E}\left\|y_{n(\theta)}+\int_{0}^{\infty} \Phi_{n(\theta)}(t) \mathrm{d} \widetilde{B}_{t}\right\|^{p} \mathrm{~d} \theta+c_{p} \delta \\
\stackrel{(\text { iii })}{\leqslant} & \frac{1}{2 \pi} \int_{0}^{2 \pi}\left(u\left(a_{n(\theta)}\right)+\varepsilon\right) \mathrm{d} \theta+c_{p} \delta \\
\stackrel{(\text { iv })}{\leqslant} & \frac{1}{2 \pi} \int_{0}^{2 \pi} u\left(x_{0}+i y_{0}+x r e^{i \theta}\right) \mathrm{d} \theta+c_{p} \delta+2 \varepsilon,
\end{aligned}
$$

where $n=n(\theta)$ is such that $a_{n}$ is closest to $x_{0}+i y_{0}+x r e^{i \theta}$ among $\left(a_{n}\right)_{n=1}^{N}$, (i) follows from the definition of $\Phi$, (ii) holds by the triangle inequality and the fact that $\left(a_{n}\right)_{n=1}^{N}$ is a $\delta$-net of $A$ (where the constant $c_{p}$ depends only on $p$ ), (iii) holds 
by (3.6), and (iv) holds by (3.5). Now if $\varepsilon \rightarrow 0$, then $\delta$ vanishes as well, and (3.4) follows.

Let us now show that $u(x) \geqslant 0$ for any $x \in X$. First, $u$ is concave in the complex variable, i.e. $y \mapsto u(x+i y)$ is concave in $y \in X$ for any $x \in X$, which follows directly from the construction of $u$ in (3.3). Now one can show that $u$ is convex in the real variable, i.e. $x \mapsto u(x+i y)$ is convex in $x \in X$ for any $y \in X$, by using the same argument as was used for plurisubharmonic functions in [24. Subsection 2.6]. Next, $u$ is symmetric, i.e. $u(x+i y)=u(-x-i y)$ for any $x, y \in X$. Thus $x \mapsto u(x)$ is a symmetric convex function with $u(0)=0$, so it is nonnegative.

(ii) $\Rightarrow$ (i). Let $u: X+i X \rightarrow \mathbb{R}$ be a function from (ii). We need to show that for any standard Brownian motions $W, \widetilde{W}: \mathbb{R}_{+} \times \Omega \rightarrow \mathbb{R}$ and for any elementary predictable process $\Phi: \mathbb{R}_{+} \times \Omega \rightarrow X$ with respect to the filtration generated by both $W$ and $\widetilde{W}$,

$$
\mathbb{E}\left\|\int_{0}^{\infty} \Phi \mathrm{d} \widetilde{W}\right\|^{p} \leqslant \chi^{p} \mathbb{E}\left\|\int_{0}^{\infty} \Phi \mathrm{d} W\right\|^{p}
$$

Since $\Phi$ is elementary predictable, it takes values in a finite-dimensional subspace of $X$, so we may assume that $X$ is finite-dimensional. Then by Subsection 2.7 we can assume that $u$ is twice differentiable on $X+i X$ by a simple convolutiontype argument. Let $d<\infty$ be the dimension of $X,\left(x_{n}\right)_{n=1}^{d}$ a basis of $X$, and $\left(x_{n}^{*}\right)_{n=1}^{d}$ the dual basis of $X^{*}$, i.e. the unique basis such that $\left\langle x_{n}, x_{m}^{*}\right\rangle=\delta_{n m}$ for any $n, m=1, \ldots, d$ (see e.g. [24], [37], [36]). Then by Itô's formula [37, Theorem 3.8] and due to local boundedness and twice differentiability of $u$ we have (here we define $M_{t}:=\int_{0}^{t} \Phi \mathrm{d} W$ and $N_{t}:=\int_{0}^{t} \Phi \mathrm{d} \widetilde{W}$ for convenience)

$$
\begin{aligned}
\chi^{p} \mathbb{E}\left\|\int_{0}^{\infty} \Phi \mathrm{d} W\right\|^{p}-\mathbb{E}\left\|\int_{0}^{\infty} \Phi \mathrm{d} \widetilde{W}\right\|^{p} \geqslant \mathbb{E} u\left(\int_{0}^{\infty} \Phi \mathrm{d} W+i \int_{0}^{\infty} \Phi \mathrm{d} \widetilde{W}\right) \\
=\mathbb{E} u\left(M_{0}+i N_{0}\right)+\mathbb{E} \int_{0}^{\infty}\left\langle\partial_{x} u\left(M_{t-}+i N_{t}\right), \mathrm{d} M_{t}\right\rangle \\
+\mathbb{E} \int_{0}^{\infty}\left\langle\partial_{i x} u\left(M_{t-}+i N_{t}\right), \mathrm{d} N_{t}\right\rangle+\frac{1}{2} \mathbb{E} I,
\end{aligned}
$$

where

$$
I=\mathbb{E} \int_{0}^{\infty} \sum_{n, m=1}^{d}\left(\frac{\partial^{2} u\left(M_{t-}+i N_{t}\right)}{\partial x_{n} x_{m}}+\frac{\partial^{2} u\left(M_{t-}+i N_{t}\right)}{\partial i x_{n} i x_{m}}\right)\left\langle\Phi, x_{n}^{*}\right\rangle \cdot\left\langle\Phi, x_{m}^{*}\right\rangle \mathrm{d} t .
$$

First notice that $\mathbb{E} u\left(M_{0}+i N_{0}\right)=\mathbb{E} u(0)=0$ and analogously to [36, proof of Theorem 3.18], $\partial_{x} u\left(M_{t-}+i N_{t}\right)$ and $\partial_{i x} u\left(M_{t-}+i N_{t}\right)$ are stochastically integrable 
with respect to $M$ and $N$ respectively, so

$$
\mathbb{E} \int_{0}^{\infty}\left\langle\partial_{x} u\left(M_{t-}+i N_{t}\right), \mathrm{d} M_{t}\right\rangle+\mathbb{E} \int_{0}^{\infty}\left\langle\partial_{i x} u\left(M_{t-}+i N_{t}\right), \mathrm{d} N_{t}\right\rangle=0,
$$

where the latter holds since both stochastic integrals are martingales which start at zero.

Let us show that $\mathbb{E} I \geqslant 0$. Fix $t \geqslant 0$ and $\omega \in \Omega$. By [37, Lemma 3.7] we are free to choose any basis (and the dual basis). In particular, we can assume that $x_{1}=\Phi(t, \omega)$. Then $\left\langle\Phi(t, \omega), x_{n}^{*}\right\rangle=\delta_{1 n}$ for any $1 \leqslant n \leqslant d$, so (here we skip $(t, \omega)$ for convenience)

$$
\begin{aligned}
& \sum_{n, m=1}^{d}\left(\frac{\partial^{2} u\left(M_{t-}+i N_{t}\right)}{\partial x_{n} x_{m}}+\frac{\partial^{2} u\left(M_{t-}+i N_{t}\right)}{\partial i x_{n} i x_{m}}\right)\left\langle\Phi, x_{n}^{*}\right\rangle \cdot\left\langle\Phi, x_{m}^{*}\right\rangle \\
& \quad=\frac{\partial^{2} u\left(M_{t-}+i N_{t}\right)}{\partial x_{1}^{2}}+\frac{\partial^{2} u\left(M_{t-}+i N_{t}\right)}{\partial i x_{1}^{2}}=\left.\Delta u\left(M_{t-}+i N_{t}+z x_{1}\right)\right|_{z=0} \geqslant 0,
\end{aligned}
$$

where $z \in \mathbb{C}$, and the last inequality follows from the diagonal plurisubharmonicity of $u$. Thus $\mathbb{E} I \geqslant 0$, and hence (3.7) follows from (3.8).

REMARK 3.2. Note that the maximum of any set of harmonic functions is harmonic as well, so the maximum of any set of diagonally plurisubharmonic functions is diagonally plurisubharmonic as well, and thus for any Banach space $X$ and for any $1<p<\infty$ with $\chi_{p, X}<\infty$ we can define an optimal diagonally plurisubharmonic function $U^{S O}: X+i X \rightarrow \mathbb{R}$ as the supremum of all functions $u$ satisfying the conditions of Proposition 3.1(ii).

Note that $U^{S O}$ coincides with the function $u$ defined by (3.3). Indeed, let $u$ be as in (3.3). Then $U^{S O} \geqslant u$ by the definition of $U^{S O}$. Let us show that $U^{S O}(x+i y) \leqslant$ $u(x+i y)$ for any $x, y \in X$. First fix independent Brownian motions $W$ and $\widetilde{W}$ and elementary predictable $\Phi: \mathbb{R}_{+} \times \Omega \rightarrow X$. Then similarly to the Itô argument from the proof of Proposition 3.1 one has

$$
U^{S O}(x+i y) \leqslant \mathbb{E} U\left(x+i y+\int_{0}^{\infty} \Phi \mathrm{d} W+i \int_{0}^{\infty} \Phi \mathrm{d} \widetilde{W}\right) .
$$

Thus

$$
\begin{aligned}
& U^{S O}(x+i y) \leqslant \inf \left\{\mathbb{E} U\left(x+i y+\int_{0}^{\infty} \Phi \mathrm{d} W+i \int_{0}^{\infty} \Phi \mathrm{d} \widetilde{W}\right):\right. \\
&\Phi \text { elementary predictable }\} \leqslant u(x+i y),
\end{aligned}
$$

as desired. 
As a corollary of Proposition 3.1 one can show the following upper and lower bounds for $\chi_{p, X}$. Recall that we define the decoupling constants $\beta_{p, X}^{\gamma,+}$ and $\beta_{p, X}^{\gamma,-}$ to be the smallest possible $\beta^{+}$and $\beta^{-}$respectively for which

$$
\frac{1}{\left(\beta^{-}\right)^{p}} \mathbb{E}\left\|\int_{0}^{\infty} \Phi \mathrm{d} W\right\|^{p} \leqslant \mathbb{E}\left\|\int_{0}^{\infty} \Phi \mathrm{d} \widetilde{W}\right\|^{p} \leqslant\left(\beta^{+}\right)^{p} \mathbb{E}\left\|\int_{0}^{\infty} \Phi \mathrm{d} W\right\|^{p}
$$

where $W$ and $\widetilde{W}$ are independent standard Brownian motions, and $\Phi: \mathbb{R}_{+} \times \Omega$ $\rightarrow X$ is elementary predictable which is independent of $\widetilde{W}$ (we refer the reader to [13], [18], [30], [21], [14], [11], [24] for further details on decoupling constants).

COROllary 3.1. Let $X$ be a Banach space, and let $1<p<\infty$. Then $\chi_{p, X}<\infty$ if and only if $X$ is UMD. Moreover, in that case

$$
\begin{aligned}
\max \left\{\sqrt{\beta_{p, X}}, \sqrt{\hbar_{p, X}}\right\} & \stackrel{(\mathrm{i})}{\leqslant} \max \left\{\beta_{p, X}^{\gamma,+}, \beta_{p, X}^{\gamma,-}\right\} \stackrel{\text { (ii) }}{\leqslant} \chi_{p, X} \\
& \stackrel{(\mathrm{iii})}{\leqslant} \min \left\{\beta_{p, X}, \hbar_{p, X}\right\} .
\end{aligned}
$$

Proof. First we show (3.9); then the "iff" statement follows. Let us first show (iii) in [3.9]. That $\chi_{p, X} \leqslant \hbar_{p, X}$ follows from [24], the definition of $\chi_{p, X}$, and the fact that any two stochastic integrals $\int \Phi \mathrm{d} W$ and $\int \Phi \mathrm{d} \widetilde{W}$ are orthogonal martingales weakly differentially subordinate to each other. The inequality $\chi_{p, X} \leqslant \beta_{p, X}$ can be proven using a standard Burkholder function argument presented e.g. in [36], [37]. Indeed, if $\beta_{p, X}<\infty$, then $X$ is UMD, and their exists a zigzag-concave function $U: X \times X \rightarrow \mathbb{R}$ (i.e. $z \mapsto U(x+z, y+\alpha z)$ is concave in $z \in X$ for any $x, y \in X$ and $\alpha \in[-1,1])$ such that $U(0,0)=0$ and

$$
U(x, y) \geqslant\|y\|^{p}-\beta_{p, X}^{p}\|x\|^{p}, \quad x, y \in X .
$$

(Such a function is called a Burkholder function.) By a standard convolution-type argument (see Subsection 2.7) we may assume that $U$ is twice differentiable, and hence for any independent standard Brownian motions $W$ and $\widetilde{W}$ and for any elementary predictable $\Phi: \mathbb{R}_{+} \times \Omega \rightarrow X$ by Itô's formula [37, Theorem 3.8] we find that analogously to (3.8), with $M:=\int \Phi \mathrm{d} W$ and $N:=\int \Phi \mathrm{d} \widetilde{W}$,

$$
\begin{aligned}
\mathbb{E}\left\|\int_{0}^{\infty} \Phi \mathrm{d} \widetilde{W}\right\|^{p}-\beta_{p, X}^{p} \mathbb{E}\left\|\int_{0}^{\infty} \Phi \mathrm{d} W\right\|^{p} \leqslant U\left(\int_{0}^{\infty} \Phi \mathrm{d} W, \int_{0}^{\infty} \Phi \mathrm{d} \widetilde{W}\right) \\
=\frac{1}{2} \int_{0}^{\infty}\left(\frac{\partial^{2} U\left(M_{t}, N_{t}\right)}{\partial(\Phi, 0)^{2}}+\frac{\partial^{2} U\left(M_{t}, N_{t}\right)}{\partial(0, \Phi)^{2}}\right) \mathrm{d} t \\
=\frac{1}{4} \int_{0}^{\infty}\left(\frac{\partial^{2} U\left(M_{t}, N_{t}\right)}{\partial(\Phi, \Phi)^{2}}+\frac{\partial^{2} U\left(M_{t}, N_{t}\right)}{\partial(\Phi,-\Phi)^{2}}\right) \mathrm{d} t \leqslant 0,
\end{aligned}
$$


where the inequality holds due to the zigzag-concavity of $U$ (so both $\frac{\partial^{2} U(x, y)}{\partial(z, z)^{2}}$ and $\frac{\partial^{2} U(x, y)}{\partial(z,-z)^{2}}$ are nonnegative for any $\left.x, y, z \in X\right)$. Thus $\chi_{p, X} \leqslant \beta_{p, X}$.

Finally, (ii) of 3.9 follows directly from the definitions of $\chi_{p, X}, \beta_{p, X}^{\gamma,+}$, and $\beta_{p, X}^{\gamma,-}$, while (i) holds by [13, p. 43 and Theorem 3].

REMARK 3.3. By the last proof, for a Burkholder function $U$, the function $-U$ is diagonally plurisubharmonic. Thus the proof of (iii) of (3.9) has the following form: both $-U$ and $U_{\mathcal{H}}$ are diagonally plurisubharmonic and thus satisfy the conditions of Proposition 3.1(ii), so the upper bound (iii) of (3.9) holds true.

In the real-valued case the functions $U^{S O}$ and $U_{\mathcal{H}}$ coincide since in this case there is no difference between plurisubharmonicity and diagonal plurisubharmonicity. If the same held for a general UMD Banach space, then we would have $\hbar_{p, X}=\chi_{p, X} \leqslant \beta_{p, X}$, which would partly solve an open problem outlined in the introduction.

\section{WEAK DIFFERENTIAL SUBORDINATION OF STRONGLY ORTHOGONAL MARTINGALES}

Now we are ready to show the main result of the paper.

THEOREM 4.1. Let $X$ be a UMD Banach space, and let $1<p<\infty$. Then for any strongly orthogonal martingales $M, N: \mathbb{R}_{+} \times \Omega \rightarrow X$ with $N \stackrel{w}{\ll} M$ one has

$$
\mathbb{E}\left\|N_{t}\right\|^{p} \leqslant \chi_{p, X}^{p} \mathbb{E}\left\|M_{t}\right\|^{p}, \quad t \geqslant 0 .
$$

Proof. By Subsection 2.7 we may assume that $X$ is finite-dimensional and that all the Bellman functions are smooth. Due to (3.2) we only need to show that

$$
\mathbb{E} U^{S O}\left(M_{t}+i N_{t}\right) \geqslant 0,
$$

where $U^{S O}$ is as in Remark 3.2. Let $d \geqslant 0$ be the dimension of $X$. Since $N \stackrel{w}{\longleftarrow} M$ and since $M$ and $N$ are orthogonal, by [24, Section 3] we know that after a proper time-change there exist a standard $2 d$-dimensional Brownian motion $W$ and predictable $\Phi, \Psi: \mathbb{R}_{+} \times \Omega \rightarrow \mathcal{L}\left(\mathbb{R}^{2 d}, X\right)$ which are stochastically integrable with respect to $W$ such that $N=\int \Psi \mathrm{d} W$ and $M=M_{0}+\int \Phi \mathrm{d} W+M^{d}$, where $M^{d}$ is purely discontinuous (see Subsection 2.2). Moreover, as $M$ and $N$ are strongly orthogonal, for any $x^{*}, y^{*} \in X^{*}$ and $t \geqslant 0$ by [20, Theorems 26.6 and 26.13] we have

$$
\left[\left\langle M, x^{*}\right\rangle,\left\langle N, y^{*}\right\rangle\right]_{t}=\int_{0}^{t}\left\langle\Phi^{*}(s) x^{*}, \Psi^{*}(s) y^{*}\right\rangle \mathrm{d} s=0 .
$$

Therefore by the Lebesgue differentiation theorem, $\left\langle\Phi^{*} x^{*}, \Psi^{*} y^{*}\right\rangle=0$ a.e. on $\mathbb{R}_{+} \times \Omega$. By choosing $\left(x^{*}, y^{*}\right)$ from a dense subset of $X^{*} \times X^{*}$ and using the fact 
that $\left(x^{*}, y^{*}\right) \mapsto\left\langle\Phi^{*} x^{*}, \Psi^{*} y^{*}\right\rangle$ is continuous on $X^{*} \times X^{*}$ on the whole $\mathbb{R}_{+} \times \Omega$, one has

$$
\left\langle\Phi^{*} x^{*}, \Psi^{*} y^{*}\right\rangle=0, \quad x^{*}, y^{*} \in X^{*},
$$

a.e. on $\mathbb{R}_{+} \times \Omega$. Furthermore, by [24, Section 3], a.s. for any $0 \leqslant s \leqslant t$ there exists a skew-symmetric operator $A(s, \omega) \in \mathcal{L}\left(\mathbb{R}^{d}\right)$ (i.e. $\langle A h, h\rangle=0$ for any $h \in \mathbb{R}^{d}$ ) of norm at most 1 such that

$$
\Psi(s, \omega)=\Phi(s, \omega) A(s, \omega) .
$$

Now let us show (4.1) using (4.2). Let $\left(x_{n}\right)_{n=1}^{d}$ be a basis of $X$, and $\left(x_{n}^{*}\right)_{n=1}^{d}$ the dual basis of $X^{*}$. By Itô's formula [37, Theorem 3.8] and smoothness of $U^{S O}$ we have

$$
\mathbb{E} U^{S O}\left(M_{t}+i N_{t}\right)=\mathbb{E} U^{S O}\left(M_{0}+i N_{0}\right)+\mathbb{E} I_{1}+\mathbb{E} I_{2}+\frac{1}{2} \mathbb{E} I_{3},
$$

where

$$
\begin{aligned}
& I_{1}=\int_{0}^{t}\left\langle\partial U^{S O}\left(M_{s-}+i N_{s}\right), \mathrm{d} M_{s}+i \mathrm{~d} N_{s}\right\rangle \\
& I_{2}=\sum_{0 \leqslant s \leqslant t}\left(\Delta U^{S O}\left(M_{s}+i N_{s}\right)-\left\langle\partial U^{S O}\left(M_{s-}+i N_{s}\right), \Delta M_{s}\right\rangle\right),
\end{aligned}
$$

and

$$
\begin{aligned}
I_{3}= & \int_{0}^{t} \sum_{n, m=1}^{d} \frac{\partial^{2} U^{S O}\left(M_{s-}+i N_{s}\right)}{\partial x_{n} x_{m}}\left\langle\Phi^{*} x_{n}^{*}, \Phi^{*} x_{m}^{*}\right\rangle \mathrm{d} t \\
& +2 \int_{0}^{t} \sum_{n, m=1}^{d} \frac{\partial^{2} U^{S O}\left(M_{s-}+i N_{s}\right)}{\partial x_{n} i x_{m}}\left\langle\Phi^{*} x_{n}^{*}, \Psi^{*} x_{m}^{*}\right\rangle \mathrm{d} t \\
& +\int_{0}^{t} \sum_{n, m=1}^{d} \frac{\partial^{2} U^{S O}\left(M_{s-}+i N_{s}\right)}{\partial i x_{n} i x_{m}}\left\langle\Psi^{*} x_{n}^{*}, \Psi^{*} x_{m}^{*}\right\rangle \mathrm{d} t .
\end{aligned}
$$

First notice that since $N_{0}=0$ and $U^{S O}(x) \geqslant 0$ for any $x \in X$, we have $\mathbb{E} U^{S O}\left(M_{0}+i N_{0}\right)=\mathbb{E} U^{S O}\left(M_{0}\right) \geqslant 0$. Moreover, $\mathbb{E} I_{1}=0$ since this is a martingale that starts at zero (which follows similarly to the proof of Proposition 3.1). Let us show that $I_{2} \geqslant 0$ a.s. Note that $x \mapsto U^{S O}(x+i y)$ is convex in $x \in X$ for any $y \in X$ by Proposition 3.1 , so by the continuity of $N$ we see that for any $0 \leqslant s \leqslant t$,

$$
U^{S O}\left(M_{s}+i N_{s}\right) \leqslant U^{S O}\left(M_{s-}+i N_{s}\right)+\left\langle\partial U^{S O}\left(M_{s-}+i N_{s}\right), \Delta M_{s}\right\rangle,
$$

and thus $I_{2} \geqslant 0$ a.s. 
Now we show that $I_{3} \geqslant 0$ a.s. To do so we need to prove that a.s., for every $0 \leqslant s \leqslant t$

$$
\begin{aligned}
\sum_{n, m=1}^{d}\left(\frac{\partial^{2} U^{S O}\left(M_{s-}+\right.}{\partial x_{n} x_{m}}\right. & \left.i N_{s}\right) \\
+ & \left.\frac{\left.\partial^{2} U^{*} x_{n}^{*}, \Phi^{*} x_{m}^{*}\right\rangle}{\partial x_{n} i x_{m}}+i N_{s}\right) \\
+ & \left.\frac{\partial^{2} U^{S O}\left(M_{s-}+i N_{s}\right)}{\partial i x_{n} i x_{m}}\left\langle\Psi_{n}^{*}, \Psi^{*} x_{m}^{*}, \Psi^{*} x_{m}^{*}\right\rangle\right) \geqslant 0 .
\end{aligned}
$$

Fix $\omega \in \Omega$ and $0 \leqslant s \leqslant t$ such that (4.2) and (4.3) hold true. Then the left-hand side of (4.4) gets the form

$$
\begin{aligned}
\sum_{n, m=1}^{d}\left(\frac{\partial^{2} U^{S O}\left(M_{s-}+i N_{s}\right)}{\partial x_{n} x_{m}}\left\langle\Phi^{*} x_{n}^{*}, \Phi^{*} x_{m}^{*}\right\rangle\right. & \\
+ & \left.\frac{\partial^{2} U^{S O}\left(M_{s-}+i N_{s}\right)}{\partial i x_{n} i x_{m}}\left\langle\Psi^{*} x_{n}^{*}, \Psi^{*} x_{m}^{*}\right\rangle\right) .
\end{aligned}
$$

Now analogously to [24, Section 3] the expression (4.5) does not depend on the choice of $\left(x_{n}\right)_{n=1}^{d}$ or, equivalently, the choice of $\left(x_{n}^{*}\right)_{n=1}^{d}$ (since one can reconstruct a basis from its dual basis [24], [37]). Moreover, by (4.3), for the symmetric nonnegative bilinear forms $V, W: X^{*} \times X^{*} \rightarrow \mathbb{R}$ defined by

$$
V\left(x^{*}, y^{*}\right):=\left\langle\Phi^{*} x^{*}, \Phi^{*} y^{*}\right\rangle, \quad W\left(x^{*}, y^{*}\right):=\left\langle\Psi^{*} x^{*}, \Psi^{*} y^{*}\right\rangle, \quad x^{*}, y^{*} \in X^{*},
$$

we see that $V\left(x^{*}, x^{*}\right)=0$ implies $W\left(x^{*}, x^{*}\right)=0$ for any $x^{*} \in X^{*}$. Thus by [24, Section 3] there exists a basis $\left(y_{n}^{*}\right)_{n=1}^{d}$ of $X^{*}$ with the corresponding dual basis $\left(y_{n}\right)_{n=1}^{d}$ of $X$, a $[0,1]$-valued sequence $\left(\lambda_{n}\right)_{n=1}^{d}$, and a number $0 \leqslant K \leqslant d$ such that $V\left(y_{n}^{*}, y_{m}^{*}\right)=\delta_{n m} \mathbf{1}_{m, n \leqslant K}$ and $W\left(y_{n}^{*}, y_{m}^{*}\right)=\lambda_{n} \delta_{n m} \mathbf{1}_{m, n \leqslant K}$ for all $m, n=1, \ldots, d$. Therefore by the discussion above we can change the basis and deduce that 4.5 ) equals

$$
\begin{aligned}
& \sum_{n, m=1}^{d}\left(\frac{\partial^{2} U^{S O}\left(M_{s-}+i N_{s}\right)}{\partial y_{n} y_{m}}\left\langle\Phi^{*} y_{n}^{*}, \Phi^{*} y_{m}^{*}\right\rangle\right. \\
&\left.+\frac{\partial^{2} U^{S O}\left(M_{s-}+i N_{s}\right)}{\partial i y_{n} i y_{m}}\left\langle\Psi^{*} y_{n}^{*}, \Psi^{*} y_{m}^{*}\right\rangle\right) \\
&=\sum_{n=1}^{K}\left(\frac{\partial^{2} U^{S O}\left(M_{s-}+i N_{s}\right)}{\partial y_{n}^{2}}+\lambda_{n} \frac{\partial^{2} U^{S O}\left(M_{s-}+i N_{s}\right)}{\partial i y_{n}^{2}}\right) .
\end{aligned}
$$

Since $y \mapsto U^{S O}(x+i y)$ is concave in $y \in X$ for any $x \in X$, it follows that $\frac{\partial^{2} U^{S O}\left(M_{s-}+i N_{s}\right)}{\partial i y_{n}^{2}} \leqslant 0$, and hence as $0 \leqslant \lambda_{n} \leqslant 1$ the right-hand side of 4.6 is 
bounded from below by (here $z \in \mathbb{C}$ )

$$
\begin{aligned}
\sum_{n=1}^{K}\left(\frac{\partial^{2} U^{S O}\left(M_{s-}+i N_{s}\right)}{\partial y_{n}^{2}}+\right. & \left.\frac{\partial^{2} U^{S O}\left(M_{s-}+i N_{s}\right)}{\partial i y_{n}^{2}}\right) \\
& =\left.\sum_{n=1}^{K} \Delta_{z} U^{S O}\left(M_{s-}+i N_{s}+z y_{n}\right)\right|_{z=0} \geqslant 0,
\end{aligned}
$$

by the diagonal plurisubharmonicity of $U^{S O}$. Therefore (4.4) holds a.e. on $\mathbb{R}_{+} \times \Omega$, and thus $\mathbb{E} I_{3} \geqslant 0$. This completes the proof of $(4.1)$ and of the theorem.

REMARK 4.1. Let $X$ be a Banach space, and $f, g: X \rightarrow \mathbb{R}_{+}$be continuous convex symmetric functions. Define $\chi_{f, g, X}$ to be the least constant $\chi \in[0, \infty]$ such that

$$
\mathbb{E} g\left(\int_{0}^{\infty} \Phi \mathrm{d} \widetilde{W}\right) \leqslant \chi \mathbb{E} f\left(\int_{0}^{\infty} \Phi \mathrm{d} W\right)
$$

for all independent Brownian motions $W$ and $\widetilde{W}$ and all elementary predictable $\Phi$. Then analogously to Theorem 4.1 we can show that $\mathbb{E} g\left(N_{t}\right) \leqslant \chi_{f, g, X} \mathbb{E} f\left(M_{t}\right)$ for any strongly orthogonal martingales $M, N: \mathbb{R}_{+} \times \Omega \rightarrow X$ satisfying $N \stackrel{w}{\ll} M$. To do so, we construct a Bellman function $U_{f, g}^{S O}$ analogously to $U^{S O}$ (see Subsection 2.7), and then proceed as in the proof of Theorem 4.1 but applying the finite-dimensional approximation described in [24]. It remains open whether weak type $L^{p}$-estimates are possible for strongly orthogonal martingales.

Acknowledgments. The author would like to thank Adam Osękowski, Mark Veraar, and the anonymous referee for helpful comments. The author thanks Stefan Geiss for fruitful discussions and for being the host during the author's stay at Jyväskylä University where the present paper was written.

\section{REFERENCES}

[1] R. Bañuelos, A. Bielaszewski, and K. Bogdan, Fourier multipliers for non-symmetric Lévy processes, in: Marcinkiewicz Centenary Volume, Banach Center Publ. 95, Inst. Math. Polish Acad. Sci., Warszawa, 2011, 9-25.

[2] R. Bañuelos and K. Bogdan, Lévy processes and Fourier multipliers, J. Funct. Anal. 250 (2007), 197-213.

[3] R. Bañuelos and A. Osękowski, Martingales and sharp bounds for Fourier multipliers, Ann. Acad. Sci. Fenn. Math. 37 (2012), 251-263.

[4] R. Bañuelos and G. Wang, Orthogonal martingales under differential subordination and applications to Riesz transforms, Illinois J. Math. 40 (1996), 678-691.

[5] J. Bourgain, Some remarks on Banach spaces in which martingale difference sequences are unconditional, Ark. Mat. 21 (1983), 163-168.

[6] J. Bourgain, On martingales transforms in finite-dimensional lattices with an appendix on the K-convexity constant, Math. Nachr. 119 (1984), 41-53.

[7] D. L. Burkholder, A geometric condition that implies the existence of certain singular integrals of Banach-space-valued functions, in: Conference on Harmonic Analysis in Honor of Antoni Zygmund (Chicago, IL, 1981), Wadsworth, Belmont, CA, 1983, 270-286. 
[8] D. L. Burkholder, Boundary value problems and sharp inequalities for martingale transforms, Ann. Probab. 12 (1984), 647-702.

[9] D. L. Burkholder, Martingales and Fourier analysis in Banach spaces, in: Probability and Analysis (Varenna, 1985), Lecture Notes in Math. 1206, Springer, Berlin, 1986, 61-108.

[10] D. L. Burkholder, Martingales and singular integrals in Banach spaces, in: Handbook of the Geometry of Banach Spaces, Vol. I, North-Holland, Amsterdam, 2001, 233-269.

[11] S. G. Cox and S. Geiss, On decoupling in Banach spaces, arXiv:1805.12377 (2018).

[12] C. Dellacherie and P.-A. Meyer, Probabilities and Potential. B, North-Holland Math. Stud. 72 , North-Holland, Amsterdam, 1982.

[13] D. J. H. Garling, Brownian motion and UMD-spaces, in: Probability and Banach Spaces (Zaragoza, 1985), Lecture Notes in Math. 1221, Springer, Berlin, 1986, 36-49.

[14] S. Geiss, A counterexample concerning the relation between decoupling constants and UMDconstants, Trans. Amer. Math. Soc. 351 (1999), 1355-1375.

[15] S. Geiss, S. Montgomery-Smith, and E. Saksman, On singular integral and martingale transforms, Trans. Amer. Math. Soc. 362 (2010), 553-575.

[16] S. Geiss and I. S. Yaroslavtsev, Dyadic and stochastic shifts and Volterra-type operators, in preparation.

[17] B. Hollenbeck, N. J. Kalton, and I. E. Verbitsky, Best constants for some operators associated with the Fourier and Hilbert transforms, Studia Math. 157 (2003), 237-278.

[18] T. P. Hytönen, J. M. A. M. van Neerven, M. C. Veraar, and L. Weis, Analysis in Banach Spaces, Vol. I. Martingales and Littlewood-Paley theory, Ergeb. Math. Grenzgeb. 63, Springer, 2016.

[19] J. Jacod and A. N. Shiryaev, Limit Theorems for Stochastic Processes, 2nd ed., Grundlehren Math. Wiss. 288, Springer, Berlin, 2003.

[20] O. Kallenberg, Foundations of Modern Probability, 2nd ed., Springer, New York, 2002.

[21] T. R. McConnell, Decoupling and stochastic integration in UMD Banach spaces, Probab. Math. Statist. 10 (1989), 283-295.

[22] J. M. A. M. van Neerven, M. C. Veraar, and L. W. Weis, Stochastic integration in UMD Banach spaces, Ann. Probab. 35 (2007), 1438-1478.

[23] A. Osękowski, Strong differential subordination and sharp inequalities for orthogonal processes, J. Theoret. Probab. 22 (2009), 837-855.

[24] A. Osękowski and I. S. Yaroslavtsev, The Hilbert transform and orthogonal martingales in Banach spaces, Int. Math. Res. Notices (online, 2019).

[25] G. Pisier, Martingales in Banach Spaces, Cambridge Stud. Adv. Math. 155, Cambridge Univ. Press, 2016.

[26] P. E. Protter, Stochastic Integration and Differential Equations, 2nd ed., Stochastic Modelling Appl. Probab. 21, Springer, Berlin, 2005.

[27] J. L. Rubio de Francia, Martingale and integral transforms of Banach space valued functions, in: Probability and Banach Spaces (Zaragoza, 1985), Lecture Notes in Math. 1221, Springer, Berlin, 1986, 195-222.

[28] M. C. Veraar, Stochastic integration in Banach spaces and applications to parabolic evolution equations, $\mathrm{PhD}$ thesis, TU Delft, 2006.

[29] M. C. Veraar, Continuous local martingales and stochastic integration in UMD Banach spaces, Stochastics 79 (2007), 601-618.

[30] M. C. Veraar, Randomized UMD Banach spaces and decoupling inequalities for stochastic integrals, Proc. Amer. Math. Soc. 135 (2007), 1477-1486.

[31] M. C. Veraar and I. S. Yaroslavtsev, Cylindrical continuous martingales and stochastic integration in infinite dimensions, Electron. J. Probab. 21 (2016), art. 59, 53 pp.

[32] G. Wang, Differential subordination and strong differential subordination for continuous-time martingales and related sharp inequalities, Ann. Probab. 23 (1995), 522-551.

[33] I. S. Yaroslavtsev, On the martingale decompositions of Gundy, Meyer, and Yoeurp in infinite dimensions, Ann. Inst. H. Poincaré Probab. Statist. 55 (2019), 1988-2018. 
[34] I. S. Yaroslavtsev, Burkholder-Davis-Gundy inequalities in UMD Banach spaces, arXiv:1807.05573 (2018).

[35] I. S. Yaroslavtsev, Even Fourier multipliers and martingale transforms in infinite dimensions, Indag. Math. (N.S.) 29 (2018), 1290-1309.

[36] I. S. Yaroslavtsev, Fourier multipliers and weak differential subordination of martingales in UMD Banach spaces, Studia Math. 243 (2018), 269-301.

[37] I. S. Yaroslavtsev, Martingale decompositions and weak differential subordination in UMD Banach spaces, Bernoulli 25 (2019), 1659-1689.

Ivan S. Yaroslavtsev

Max Planck Institute for Mathematics in the Sciences

Inselstraße 22

04103 Leipzig, Germany

E-mail: yaroslavtsev.i.s@yandex.ru

Received 12.2.2019;

revised version 5.5.2020 
